\title{
Design of PID Controller for LQR Method in Direct Drive DC Motor
}

\author{
Sang-Min Lee ${ }^{1}$, Houng-Kun Joung ${ }^{2}$ and ${ }^{*}$ Nam Kim$^{1}$ (Corresponding Author) \\ ${ }^{1}$ Dept. of Telecommunication Engineering, Chungbuk Narional University \\ ${ }^{2}$ Dept. of Electrical Engineering, Hanyang University \\ ${ }^{1}$ leesm@cbtp.or.kr, ${ }^{2} 21 c 8545 @$ hanyang.ac.kr, ${ }^{1}$ namkim@chunbuk,.ac.kr
}

\begin{abstract}
A Direct Drive DC Motor is directly connected to a motor without the use of a belt or gear. Thus, it can compensate for the shortcomings incurred by the heat of gears which induces nonlinear characteristics such as backlash, friction, and dead zone. However, direct acting has a great sensitivity because it causes load inertia to bring about uncertainty. In this regard, this paper propose for addressing the uncertainty that induces the changes in load inertia of the Direct Drive DC Motor by designing a PID Controller using LQR Method
\end{abstract}

Keywords: Direct Drive DC Motor, PID Controller, LQR, Loop Shaping

\section{Introduction}

Unlike a general DC motor, the Direct Drive DC Motor has a high acceleration torque by connecting a load directly to a motor shaft, has no nonlinear characteristics such as twist or friction due to the heat of gears. In addition, it was miniaturized by the development of new material and widely used in high precision robot application field, prompting active researches on it. However, having no heat in its gears, the motor gets highly sensitive to the changes in the load inertia. To compensate for these changes, Butler [1] proposed a Model Reference Adaptive Control to make up for the changing load inertia, and Hamdi's [2] fuzzy controller takes nonlinear characteristics into consideration and has an advantage of requiring no complex mathematical modeling. The adaptive controllers of Kim [3] and $\mathrm{Xu}$ [4] compensated the steady state position errors using the forward compensator with state feedback and enhanced the command following performance using the auxiliary input. Furuta [5] designed a Bang-bang Controller using VSS (Variable Structure System) -type integrator, which led to the guaranty for their robustness against parameter changes and model errors.

This paper proposes a PID Controller [6] using LQR Method as a Position Controller of the Direct Drive DC Motor, which can solve the issue of the load speed getting more sensitive due to the uncertainty incurred by the load inertia and the difficulty of controlling the motor and secure the robustness against the errors arising when controlling the position of the motor.

This controller, which has LQR State Feedback and PID Controller Output Feedback at the same time, has improved the position following and disturbance rejecting performance by using Loop Shaping to limit Loop Transfer Function values in low and high frequency regions and by giving weighted values to each of the regions

\section{Structure of the Direct Drive DC Motor}

Equation (1) and (2) shows the standard model transfer function of a DC motor, which is composed of an electrical transfer function and a mechanical transfer function. 


$$
\begin{gathered}
E(s)=\frac{1}{s L_{m}+R_{a}} \\
M(s)=\frac{1}{s J_{t}+f}
\end{gathered}
$$

Coulomb friction by magnetic and mechanical hysteresis is the result of a reaction torque having the same sign and magnitude $\left(M_{\omega}\right)$ as velocity $\left({ }^{\omega_{p}}\right)$. This static model only approximates the actual friction effect. In addition, the current loop can be approximated to 1 because it is compared with the time constant of the motor when operating at high speed.

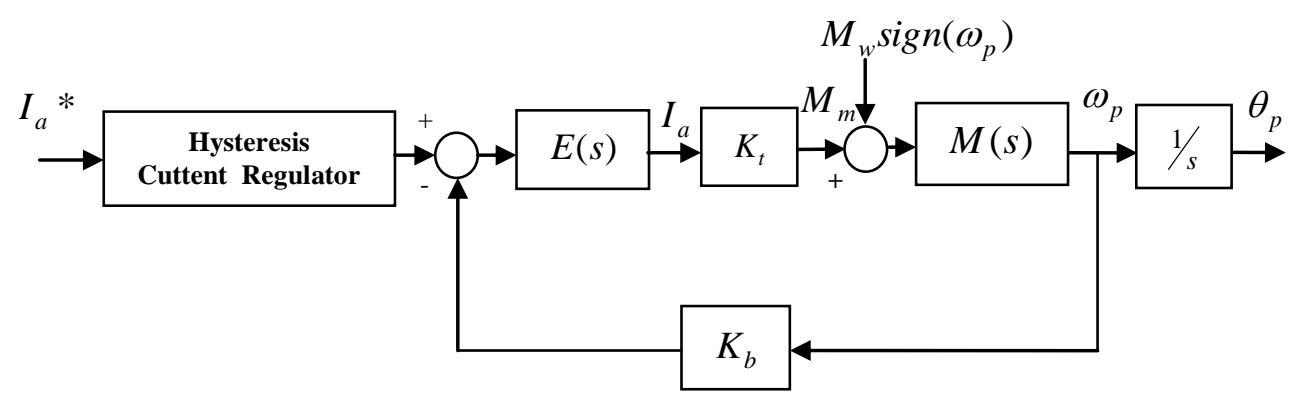

Figure 1. Block Diagram of DC Motor

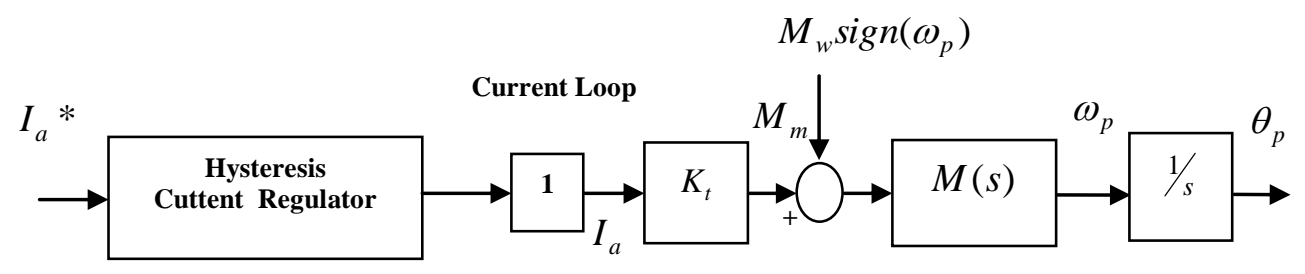

Figure 2. Block Diagram of DC Motor \& Current Loop

\begin{tabular}{l|l|l|l}
\hline$R_{a}$ & Resistance & $J t$ & Overall inertia moment \\
\hline$L_{m}$ & Motor Inductance & $M_{\omega}$ & Coulomb friction \\
\hline$K_{t}$ & Torque constant & $f$ & Braking \\
\hline$I_{a}$ & Current & $K_{b}$ & Back electromotive force constant \\
\hline
\end{tabular}

\section{Controller Design}

The basic differential equation of the Direct Drive DC Motor is shown in equation (3).

$$
J_{t} \frac{d \theta_{p}^{2}}{d^{2} t}+f \frac{d \theta_{p}}{d t}=K_{t} I_{a}
$$

where, $\theta_{p}$ is the output variable and $l_{a}$ is the control variable $u(t)$.

Since the LQR control system is structured in the way that the error signal cannot be controlled because it is not inside the feedback loop, an inclusion of the output variable as the state variable is required to designing the controller with excellent command following performance. Therefore, state space model is obtained as equation (4) if the 
definition is made by including derivative variables of state and output variables.

$$
\left\{\begin{array}{c}
\frac{d x(t)}{d t}=A_{p} x(t)+B_{p} u(t) \\
y_{p}(t)=C_{p} x(t)
\end{array}, \quad A_{p}=\left[\begin{array}{ll}
0 & 1 \\
0 & \frac{f}{J_{t}}
\end{array}\right], \quad B_{p}=\left[\begin{array}{c}
0 \\
\frac{K_{t}}{J_{t}}
\end{array}\right], \quad c_{p}=\left[\begin{array}{l}
1 \\
0
\end{array}\right]\right.
$$

Equation (6) shows the process of generating an augmented state-space description by adding integral control elements as state variables to the feed forward loop to generate a PID structure as in Equation (5).

$$
\begin{gathered}
x(t)=\left[\begin{array}{c}
\int_{t}^{0} y(\imath) d \imath \\
y(t) \\
\frac{d y(t)}{d t}
\end{array}\right]=\left[\begin{array}{c}
x_{0}(t) \\
x_{1}(t) \\
x_{2}(t)
\end{array}\right] \\
\frac{d x(t)}{d t}=A x(t)+B u(t), \quad A=\left[\begin{array}{lll}
0 & 1 & 0 \\
0 & 0 & 1 \\
0 & \frac{f}{J_{t}} & 0
\end{array}\right], B=\left[\begin{array}{c}
0 \\
0 \\
\frac{K_{t}}{J_{t}}
\end{array}\right], \quad c=\left[\begin{array}{l}
0 \\
1 \\
0
\end{array}\right]
\end{gathered}
$$

Where, the weighted matrix $\mathrm{Q}$ is $Q=N^{\top} N, R=\rho /$, and a positive semi-limited symmetric matrix. The weighted matrix $R$ is a positive definite symmetric matrix. The $\mathrm{LQR}$ problem is the problem of obtaining the design parameters $\mathrm{Q}$ and $\mathrm{R}$ that minimize the price function of equation (7), and the optimal control input is obtained as in equation (8) [7].

$$
u(t)=-G x(t), G=-R^{-1} B^{T} K
$$

where, $\mathrm{K}$ is the solution of the Riccati equation of equation (9).

$$
K A+A^{T} K+Q-K B R^{-1} B^{T} K=0
$$

The control gain matrix $\mathrm{G}$ can be decomposed as equation (10).

$$
G=\left[\begin{array}{lll}
g_{0} & g_{1} & g_{2}
\end{array}\right]
$$

Therefore, equation (8) is expressed in the form of PID control as in equation (11) using equation (6).

$$
u(t)=-\left(g_{0} \int_{0}^{t} y(\tau) d \tau+g_{1} y(t)+g_{2} \frac{d y(t)}{d t}\right)
$$

The form of the PID controller of the Direct Drive DC Motor can be expressed as Equation (12).

$$
u(t)=-K_{p}\left(y(t)+\frac{1}{T_{i}} \int_{0}^{t} y(\tau) d \tau+T_{d} \frac{d y(t)}{d t}\right)
$$

Equation (13) shows the gain element $g_{0}, g_{1}, g_{2}$ of each part of the control gain matrix in equation (11) represented by the relationship between the proportional gain $K_{p}$, the integral element time ${ }^{T_{i}}$, and the derivative element time ${ }^{T_{d}}$ in the equation (12).

$$
K_{p}=g_{1}, T_{i}=\frac{g_{1}}{g_{0}}, T_{d}=\frac{g_{2}}{g_{1}}
$$




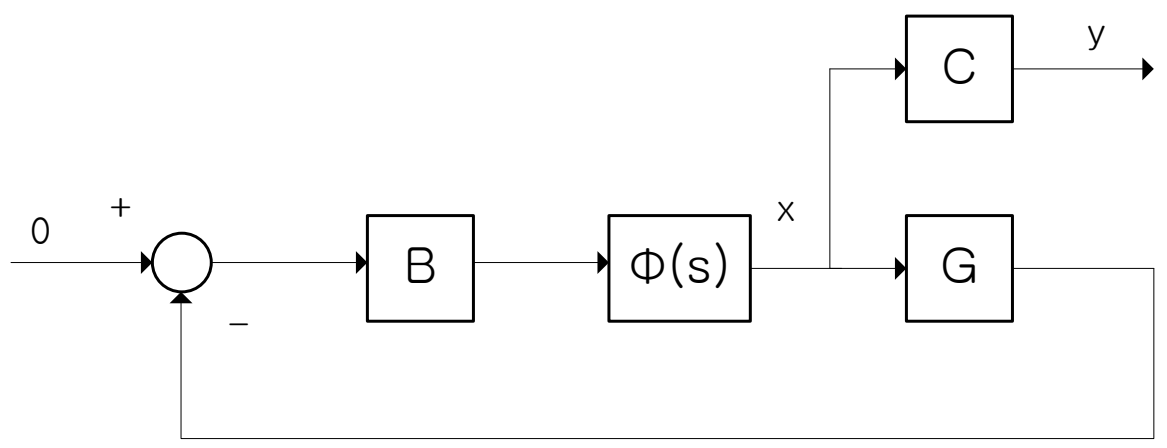

Figure 3. Block Diagram of LQR

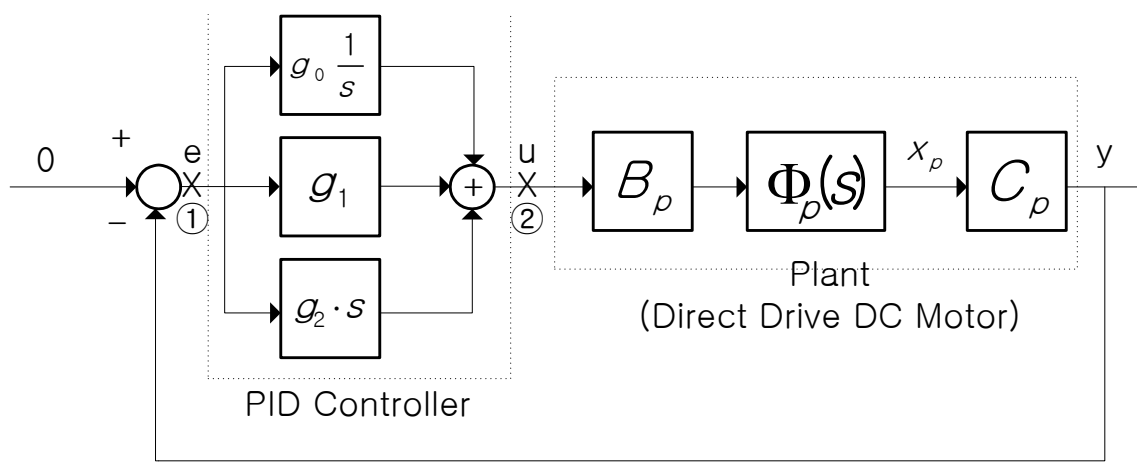

Figure 4. Block Diagram of PID Structure

In the case of a secondary system in an affixation, the LQR structure in Figure 3 and the PID structure in Figure become an equivalence. Therefore, the parameters of the PID controller can be obtained by solving the LQR design problem.

The selection of the design parameters $\mathrm{Q}$ and $\mathrm{R}$ should be made by using the singular value matching technique ${ }^{[8]}$ of the loop transfer function in order to improve the robustness of the performance. To deal with the performance problem with the loop forming technique, the loop transfer function $h(s)$ obtained from the cut point (1) of the plant in Figure 4 and the loop transfer function $g_{L}(s)$ obtained from cut point (2). $h(s)$ and $g(s)$ are expressed as equations (14) and (15).

$$
\begin{aligned}
& h(s)=C_{p}\left(s /-A_{p}\right)^{-1} B_{p}\left(g_{2} s+g_{1}+\frac{g_{0}}{s}\right)=\frac{g_{2} s^{2}+g_{1} s+g_{0}}{s\left(J_{t} s^{2}+K, s\right)} \\
& g(s)=\left(g_{2} s+g_{1}+\frac{g_{0}}{s}\right) C_{p}\left(s /-A_{p}\right)^{-1} B_{p}=\frac{g_{2} s^{2}+g_{1} s+g_{0}}{s\left(J_{t} s^{2}+K_{r} s\right)}
\end{aligned}
$$

In a single input single output (SISO) system, $h(s)$ and $g(s)$ are identical and it can be seen that they are also identical to the loop transfer function $g_{L Q}(s)$ of LQR as in Equation (16). 


$$
\begin{aligned}
g_{L Q}(s) & =G(s /-A)^{-1} B=\left[\begin{array}{lll}
g_{0} & g_{1} & g_{2}
\end{array}\right]\left[\begin{array}{cc}
\frac{1}{s} & \frac{1}{s} C_{p}\left(s /-A_{p}\right)^{-1} \\
0 & \left(s /-A_{p}\right)^{-1}
\end{array}\right] \\
& =\frac{1}{s} g_{0} C_{p}\left(s /-A_{p}\right)^{-1} B_{p}+\left[\begin{array}{ll}
g_{1} & g_{2}
\end{array}\right]\left(s /-A_{p}\right)^{-1} B_{p} \\
& =\frac{g_{2} s^{2}+g_{1} s+g_{0}}{s\left(J_{t} s^{2}+K, s\right)}
\end{aligned}
$$

Design parameters $Q=N^{T} N$ and $R=\rho /$ can be determined in Equation (17), using the frequency domain equality of the LQ problem,

$$
\left[1+g_{L Q}(-j \omega)\right] R\left[1+g_{L Q}(-j \omega)\right]=R+g_{O L}(-j \omega) g_{O L}(j \omega)
$$

where, $g_{L Q}(j \omega)=G(j \omega /-A)^{-1} B$ and $g_{O L}(j \omega)$ is an open loop transfer functions $g_{O L}(j \omega)=N(j \omega /-A)^{-1} B$. As $h(s)$ and $g_{L Q}(s)$ are identical in the SISO system, the inverse frequency equation (17) can be rewritten as Equation (18).

$$
[1+h(-j \omega)]\left[R[1+h(j \omega)]=R+g_{O L}(-j \omega) g_{O L}(j \omega)\right.
$$

The inverse frequency characteristic of LQR can be expressed as Equation (19) from Equation (18).

$$
|/+h(j \omega)|=\sqrt{1+\frac{1}{\rho}\left|N(j \omega /-A)^{-1} B\right|^{2}}
$$

where, the design parameter $\mathrm{N}$ can be decomposed as in equation (20).

$$
N=\left[\begin{array}{lll}
n_{0} & n_{1} & n_{2}
\end{array}\right]
$$

The frequency domain performance is generally expressed by system performance such as Command Following, Disturbance Rejection, and insensitivity to sensor noise and higher order modeling error reduction. Because Command Following is a function that enables the output of the system to well follow the reference input, the command following performance should be made good only in the low frequency range where the reference input has energy. In addition, since the plant plays a role of a low-pass filter, the disturbance reflected on the output side of the Direct Drive DC Motor mostly has energy at a low frequency. Therefore, the performance of Disturbance Rejection is also important in at a low frequency range. Generally, the sensor noise and the high order modeling errors have energy at a high frequency range, so their reducing performance should be made work well only at a high frequency range. Therefore, it is possible to make a design which can satisfy all of the following performances: command following, disturbance rejecting, and low sensitivity to sensor noises, in consideration of each frequency region where the reference input, disturbance, sensor noises, and high-order modeling errors have energy.

In this paper, there are always two poles in the PID design method for controlling the Direct Drive DC Motor. And the two pole points of the Direct Drive DC Motor are all real roots rather than complex Conjugates.

If the two pole points of the Direct Drive DC Motor are defined as $p_{1}$ and $p_{2}$ respectively with ${ }^{p_{2}}$ defined as the maximum pole point, the relationship between the two pole-points is expressed by Equation (21).

$$
\left|p_{2}\right| \geq\left|p_{1}\right|
$$


In the PID control system, the two poles of the Direct Drive DC Motor and the loop transfer function $|h(j \omega)|$ are identical, so $p_{1}$ and $p_{2}$ become the pole point of the loop transfer function $|h(j \omega)|$.In addition, if the zero of $|h(j \omega)|$ is defined as $z_{1}$ and $z_{2}$, with Equation (19) approximated and applied to the direct drive DC in the low frequency region, the process can be expressed by Equation (22).

$$
|h(j \omega)| \approx \frac{1}{\sqrt{\rho}}\left|\frac{n_{2} K_{t}\left(s-z_{1}\right)\left(s-z_{2}\right)}{s\left(s-p_{1}\right)\left(s-p_{2}\right)}\right| \quad, \quad \omega\left\langle\left\langle\omega_{c}\right.\right.
$$

In the high-frequency domain, it can be expressed by Equation (23).

$$
|h(j \omega)| \leq|\tilde{h}(j \omega)|=\frac{n_{2} K_{t}}{\sqrt{\rho}}\left|\frac{\left(s-z_{1}\right)\left(s-z_{2}\right)}{s\left(s-p_{1}\right)\left(s-p_{2}\right)}\right| \quad, \quad \omega<\left\langle\omega_{c}\right.
$$

Because $p_{1}$ and $p_{2}$, the poles of $|h(j \omega)|$ are given by the model of the Direct Drive DC motor, the position of the pole on the Bode Plot becomes fixed to a given design specification. However, the zeroes of $|h(j \omega)|$ or a $|\tilde{h}(j \omega)|$ can be shifted depending on the values of the design parameter $n_{0}, n_{1}, n_{2}$.

Depending on the positions of Pole ${ }^{p_{1}}$, which is determined by the design specification of the Direct Drive DC Motor, and Zero ${ }^{z_{1}}$ and $z_{2}$ which can be shifted along with ${ }^{p_{2}}$, the loop shape of $|h(j \omega)|$ or $|\tilde{h}(j \omega)|$ changes as in Figure 5. 


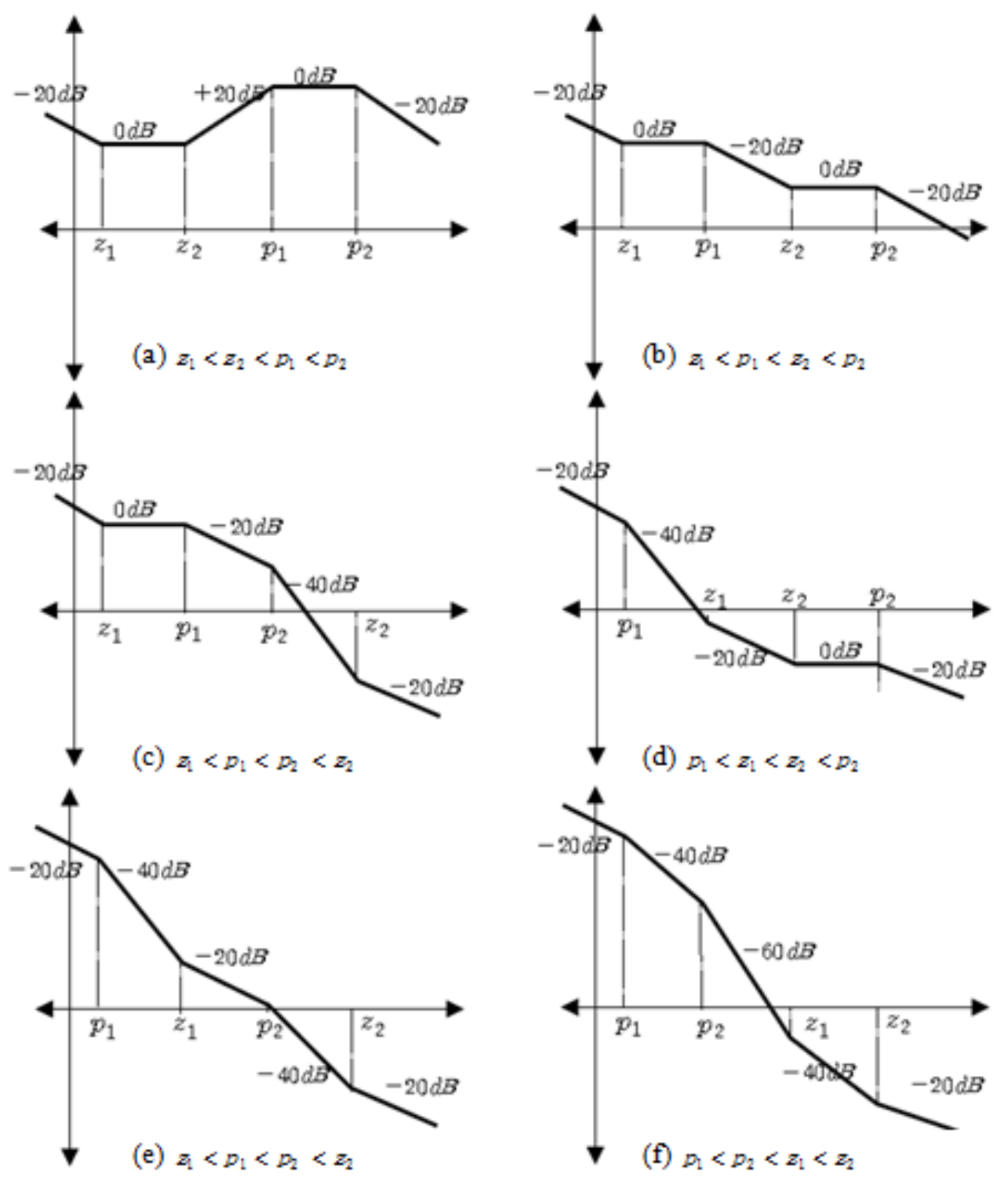

Figure 5. Bode Plot of ${ }^{|h(j \omega)|}$ According Changes of Zeros

Considering the simple characteristics of the Bode diagram [24], where the slope of $|h(j \omega)|$

at the pole position is reduced by $-20 \mathrm{~dB} / \mathrm{dec}$ from the existing slope, while the slope at the zero point is increased by $20 \mathrm{~dB} / \mathrm{dec}$, it can be easily understood that the loop shape changes depending on the positions of the pole and zero. In Figure 5, (f) represents the most desirable loop shape, which has the highest gain at a low frequency range and the lowest gain at a high frequency range. In (f) in Figure 5,the farther away Zero $z_{1}$ and $z_{2}$ from the plant maximum pole, the lower the gain at a high-frequency range. However, the zero point shifted using the PID controller cannot be shifted indefinitely because it needs more energy as it is moved further away from the origin point. The Direct Drive DC Motor is a secondary system, and the degree of roll-off at a high-frequency range is set to $-20 \mathrm{~dB} / \mathrm{dec}$ when the PID controller is added. The larger the roll-off, the better the noise rejection performance. However, as shown in (f) in Figure 5, no matter how far the zero point is from the maximum pole, the roll-off will eventually be $-20 \mathrm{~dB} / \mathrm{dec}$. 
Therefore, the case where the maximum pole of the plant ${ }^{p_{2}}$ and the two zero points to be shifted are identical is the most efficient case in (f) of Figure 5. And Equation (22) and Equation (23) can be expressed using the limits behavior ${ }^{[9]}$ as in equation (24).

$$
\lim _{z_{1}, z_{2} \rightarrow p_{2}} \mid h\left(j \omega\left|\approx \frac{n_{2} K_{t}}{\sqrt{\rho}}\right| \frac{\left(s^{2}-2 p_{2} s+p_{2}^{2}\right)}{s\left(s-p_{1}\right)\left(s-p_{2}\right)} \mid\right.
$$

Through the comparison of the coefficients of Equations (22), (23) and (24), the correlation between the design parameters $n_{0}, n_{1}$, and $n_{2}$ are calculated as in Equation (25).

$$
N=\left[\begin{array}{lll}
n_{0} & n_{1} & n_{2}
\end{array}\right]=\left[\begin{array}{lll}
n_{2} p_{2}^{2} & -2 n_{2} p_{2} & n_{2}
\end{array}\right]
$$

The minimum boundary values that satisfy the command following and disturbance rejection obtained by using the relationship seen in Equation (25) is Equation, and Equation (27) is the summary of the calculation process.

$$
\begin{gathered}
\lim _{z_{1}, z_{2} \rightarrow p_{2}}|h(j \omega)| \approx \frac{n_{2} K_{t}}{\sqrt{\rho}}\left|\frac{\left(\Omega_{r}\right)^{2}-2 p_{2}\left(\Omega_{r}\right)+p_{2}^{2}}{\left(\Omega_{r}\right)\left(\Omega_{r}-p_{1}\right)\left(\Omega_{r}-p_{2}\right)}\right|>m_{r} \\
\frac{n_{2} K_{t}}{\sqrt{\rho}}\left|\frac{\Omega_{r}-p_{2}}{\left(\Omega_{r}\right)\left(\Omega_{r}-p_{1}\right)}\right|>m_{r}
\end{gathered}
$$

where, $\Omega_{r}$ is the boundary frequency of the barrier for command following and disturbance rejection, and $m_{r}$ is the barrier for command following and disturbance rejection.

The maximum boundary value satisfying the sensor noise barrier is expressed by Equation (28), which is summarized as in Equation (29)

$$
\begin{gathered}
\lim _{z_{1}, z_{2} \rightarrow p_{2}}\left|\tilde{h}\left(\Omega_{n}\right)\right|=\frac{n_{2} K_{t}}{\sqrt{\rho}}\left|\frac{\left(\Omega_{n}\right)^{2}-2 p_{2}\left(\Omega_{n}\right)+p_{2}^{2}}{\left(\Omega_{n}\right)\left(\Omega_{n}-p_{1}\right)\left(\Omega_{n}-p_{2}\right)}\right|>e_{\max }^{-1}\left(\Omega_{n}\right) \\
\frac{n_{2} K_{t}}{\sqrt{\rho}}\left|\frac{\Omega_{n}-p_{2}}{\left(\Omega_{n}\right)\left(\Omega_{n}-p_{1}\right)}\right|>e_{\max }^{-1}\left(\Omega_{n}\right)
\end{gathered}
$$

where, $\Omega_{n}$ is the boundary frequency of the sensor noise barrier, ${ }^{-1}{ }_{\max }^{-1}$ is the barrier of modeling error. In addition, the area of $n_{2}$ that satisfies both Equation (27) and (29) from the given Direct Drive DC Motor and design specifications can be expressed by Equation (30).

$$
\frac{\mid\left(\Omega_{r}\right)\left(\Omega_{r}-p_{1)} \mid m_{r} \sqrt{\rho}\right.}{\left|\omega_{n}^{2}\left(\Omega_{r}-p_{2}\right)\right|}<n_{2}<\frac{\mid\left(\Omega_{r}\right)\left(\Omega_{r}-p_{1)} \mid e_{\max }^{-1}\left(\Omega_{n}\right) \sqrt{\rho}\right.}{\mid \omega_{n}^{2}\left(\Omega_{r}-p_{2} \mid\right.}
$$




\section{Simulation}

The following are the design specifications of the Direct Drive DC Motor to be used in the simulation.

\begin{tabular}{|c|l|}
\hline \multicolumn{2}{|c|}{ System Parameter } \\
\hline$J_{t}$ & $0.210^{\left[\mathrm{Nms}^{2} / \mathrm{rad}\right]}$ \\
\hline$f$ & $0.013^{\left[\mathrm{Nms}^{2} / \mathrm{rad}\right]}$ \\
\hline$K_{t}$ & $3.26^{[\mathrm{Nm} / \mathrm{A}]}$ \\
\hline$M_{r}$ & $100^{[\mathrm{dB}]}$ \\
\hline$e^{\max }$ & $\omega / 10^{3}$ \\
\hline
\end{tabular}

Boundary values of command following and disturbance barrier: $\Omega_{r}=10^{-1}$ Boundary values of sensor noise barrier: $\Omega_{n}=10^{-3}$

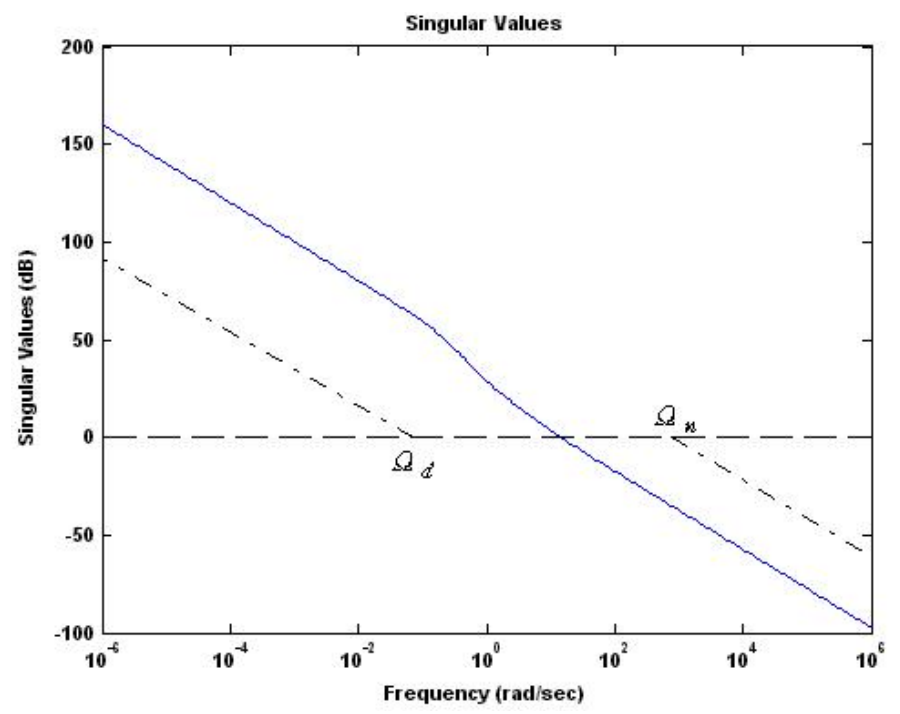

Figure 6. Singular Values of Loop Transfer Function 


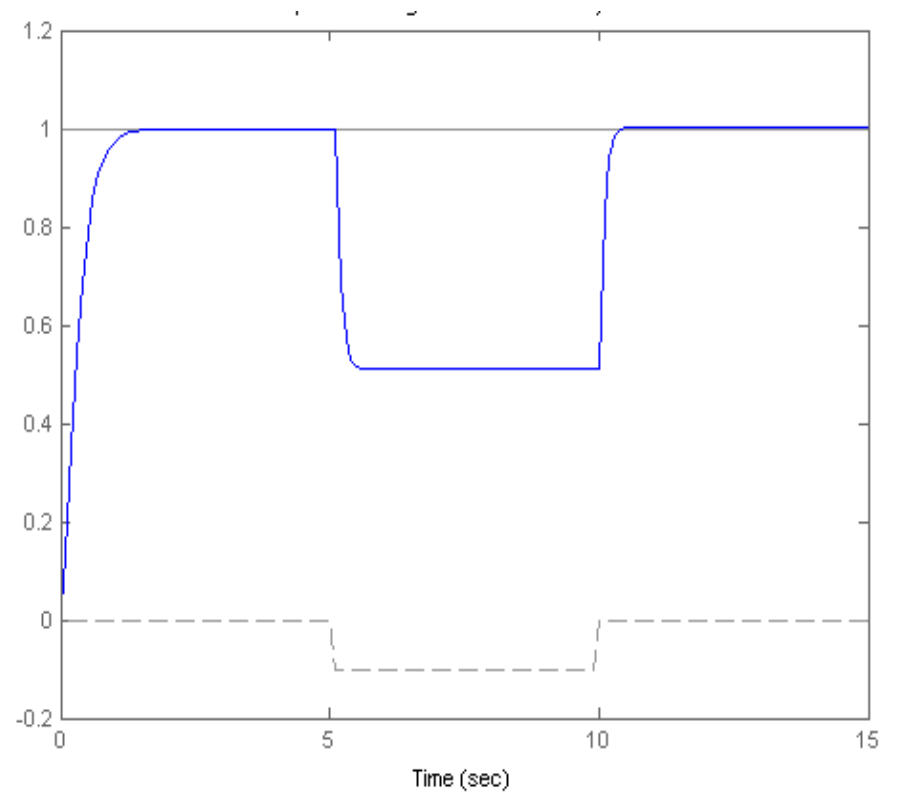

Figure 7. Pulse Response of Direct Drive DC Motor

The following shows the area of $n_{2}$ that satisfies the design conditions to obtain the design parameter $\mathrm{N}$ using the method presented in this paper,

$$
4.2011 \leq n_{2} \leq 21.41, \quad n_{2}=5
$$

The design parameter $\mathrm{N}$ and the control gain matrix $\mathrm{G}$ are obtained from the control weighting matrix $R=\rho /(\rho=1)$ as follows.

$$
N=\left[\begin{array}{llll}
501.1 & 103.4 & 5
\end{array}\right] G=\left[\begin{array}{llll}
501.1 & 153.4 & 11.5
\end{array}\right]
$$

Figure 6 shows the singular values of the loop transfer function, which guarantee the frequency domain performance-robustness in the plant presented by Yun and Suh [12].

That is, it can be seen that the curve of the loop transfer function does not impair the command following barrier and disturbance barrier at the low frequency $\left(\Omega_{r}\right)$ and the sensor noise barrier at the high frequency $\left(\Omega_{r}\right)$

As shown in Figure 7, introducing the weighted value to each frequency domain improved the rise time and the settling time, and an increase in the gain in the low frequency domain enhanced the performance of command following and disturbance rejection.

\section{Conclusion}

Using the design method of the LQ- PID controller, this paper secured the robustness against the errors incurred by the uncertainty of system due to the changes in the load inertia of the Direct Drive DC Motor when tracking the position of the motor. In addition, it demonstrated that the performance of command following and disturbance rejection can be improved by the increased gain obtained by giving the weighted values to the lowfrequency domain. Therefore, the significance of this study lies in the fact that it made an attempt to solve the design complexity and realization difficulty of the existing controllers such as adaptive controller by designing the LQ-PID controller and incorporate it into the PID structure which is widely used in industry. 


\section{References}

[1] H.Butler, G.Hoderd.and J.VAmerongen, "Model Reference Adaptive Control of a Direct Drive DC Motor", IEEE Control Systems Magazine, Vol. 1, Issue 1, pp. 80-84, Jan, 1989.

[2] Katsuhisa Furuta, Seiichi Kobayashi, "Bang-Bang Position Control of Direct Drive Motor", IECON'90, $16^{\text {th }}$ Annual conference of IEEE, Vol 1, pp. 148-153, 27-90, Nov, 1990.

[3] Hoang Le-Huy and Maher Hamdi, "Control of a Direct Drive Motor by Fuzzy Logic", Industry Application Society Annual Meeting, 1993., Conference Record of the 1993 IEEE, Vol 1, pp. 732-738, 2-8, Oct, 1993.

[4] Young T .Kim, M. R. Akbarzadeh-Tand Dong Wook Lee, "State Feedback Adaptive Control of a Direct Drive Motor", IEEE System Theory, Proceeding of the Twenty-Ninth Southeastern Symposium on, pp. 288-291, 1997.

[5] J. D. Han, Y. C. Wang, D. L. Tan, and W. L. Xu, " Acceleration Feedback Control for Direct-Drive Motor system", Intelligent Robots and System, Proceeding. 2000 IEEE/RSJ International Conference on, Vol 2, pp. 1068-1074, 31, Oct, 2000.

[6] Seong-O Yun and Byung-Suhl Suh "A New Loop Shaping Method for Design of Robust Optimal PID Controller" KICS '03-11 Vol.28 No.11C, 2003

[7] Athans M., Lecture Note on Multivariable Control System, M.I.T. Ref. No.860224/6234, 1986

[8] Seong-O Yun and Byung-Suhl Suh, "LQ-Servo Design to Command Following and Output-Disturbance Rejection", Journal of control Automation and Systems Engineering, Vol. 3, No. 5, October. 1997

[9] H. Kwakernaak and R. Sivan, “ The Maximally Achievable Accuracy of Linear Optimal Regulators and Linear Optimal Filters”, IEEE Trans, Automat. Contr., Vol. AC-17, pp.79-86, 1972. 4, pp.689-702, (1987)

\section{Authors}

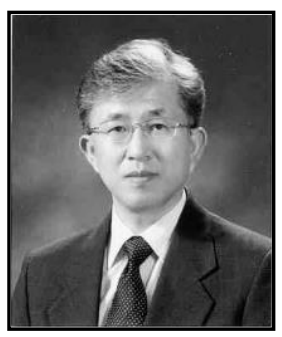

Nam Kim, received the $\mathrm{BS}$, $\mathrm{MS}$ and $\mathrm{PhD}$ degrees in the electronics engineering from Yonsei University, Seoul, Korea, in 1981, 1983, and 1988 respectively. He is a professor in the college of electrical and computer engineering, Chungbuk National University from 1989. His scientific interests are focused on the health effect of the EMF, RF dosimetry and SAR, the reduction and protection technology of the EMF hazards, and the guideline and standards on the EMF. He joined the Stanford University as a visiting professor during 1992-1993 and also visited the Caltech as an associate researcher during 2000-2001.

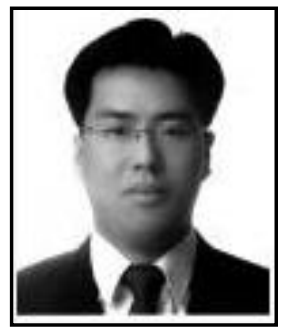

Sangmin Lee, received the BS and MS degrees in the electronics engineering from Chungbuk National University, Changju, Korea, in 2002 and 2010 respectively. He is a enginner EMC(electromagnetic compatibility) and reliability evaluation at ChungbukTechnopark from 2005. His scientific interests are focused on the electromagnetic compatibility and reliability evaluation technology the guideline standards

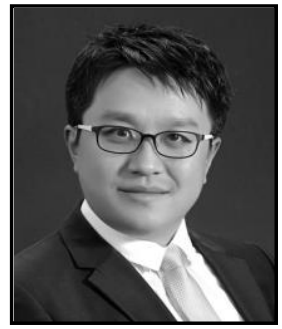

Houngkun Joung, received the BS degrees in the electrical engineering from Semyung University, Seoul, Korea, in 2002. and the MS and $\mathrm{PhD}$ degrees in the electrical engineering from Hanyang university, Seoul, Korea, in 2004, 2015 respectively. He is a associate professor in the electrical control engineering, Korea Polytechnic college from 2011. 
International Journal of Control and Automation Vol.10, No.4 (2017) 